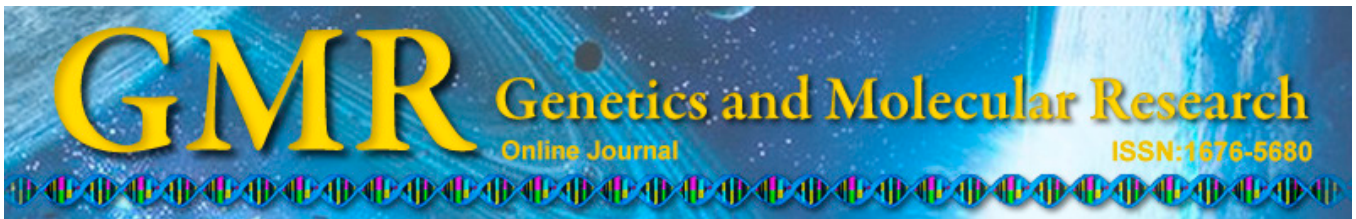

\title{
A study of the association between the connexin 40 rs35594137 polymorphism and atrial fibrillation in Xinjiang Chinese Han and Uygur populations
}

\author{
Y. Feng ${ }^{1 *}$, J. Sun ${ }^{2 *}$, L. Wang ${ }^{1}$ and X.L. Hou ${ }^{1}$ \\ ${ }^{1}$ Department of Electrocardiography, \\ People's Hospital of Xinjiang Uygur Autonomous Region, Urumqi, Xinjiang, \\ China \\ ${ }^{2}$ Cardiac Center, The First Affiliated Hospital of Xinjiang Medical University, \\ Urumqi, Xinjiang, China \\ *Theses author contributed equally to this study. \\ Corresponding author: Y. Feng \\ E-mail: 454726435@qq.com
}

Genet. Mol. Res. 14 (4): 15705-15712 (2015)

Received July 12, 2015

Accepted October 7, 2015

Published December 1, 2015

DOI http://dx.doi.org/10.4238/2015.December.1.22

\begin{abstract}
Atrial fibrillation (AF) occurrence has a known genetic component. Many reports have revealed a correlation between gene mutation and AF, involving genes related to ion channels, connexin $(\mathrm{Cx})$, and those within the angiotensin system. In this study, the correlation between the $\mathrm{Cx} 40$ polymorphism (rs35594137) and AF was investigated in patients with AF in the Xinjiang, Turpan, and Kashi regions and in controls. The AF cohort included 122 patients (58 Han and 64 Uygur). The control subjects were recruited according to the 1:1 corresponding method and matched for age and gender. Polymerase chain reaction-restriction enzyme fragment length polymorphism was used to analyze Cx 40 (rs35594137) genotype and allele distributions. Compared with the control group, A allele and A allele frequency were
\end{abstract}


higher in the promoter region of $\mathrm{Cx} 40$ (rs35594137) site $(\mathrm{P}<0.05)$. In the Uygur population, the distribution of rs35594137 genotype and allele frequencies was not significantly different $(\mathrm{P}>0.05)$ from the AF and control groups of Chinese Han origin. The confounding factors, including coronary heart disease, hypertension, smoking, and drinking, were evaluated by multivariate conditional logistic regression analysis. Cx 40 (rs35594137) differences between AF and control groups of Han origin were not significant $(\mathrm{P}>0.05)$, but were statistically significant in the Uygur population $(\mathrm{P}<0.05)$. These results demonstrated that $\mathrm{Cx}$ 40 (rs35594137) was associated with AF. In the Uygur population, Cx 40 (rs35594137) should be considered as an independent risk factor for patients with AF, who might have racial differences in rs35594137 variant frequencies.

Key words: Atrial fibrillation; Gene polymorphism; rs35594137; Connexin $40(\mathrm{Cx} 40)$

\section{INTRODUCTION}

Atrial fibrillation (AF) is the most common type of rapid arrhythmia. In China, the morbidity rate of adults with $\mathrm{AF}$ is about $0.77 \%$. The occurrence mechanisms of AF include pathogenic factors acting on multiple aspects of the electrophysiological, mechanical, and molecular biological mechanisms, among others. Although many studies in China and abroad have investigated the etiology of AF, these mechanisms remain poorly understood. In recent years, molecular genetic mechanisms have received considerable attention and an increasing amount of genetic evidence has been associated with the occurrence of AF. Many reports have also unveiled the correlation between gene mutation and AF, involving genes related to ion channels, connexin (Cx), and those in the angiotensin system, among others (Lai et al., 2002; Schreieck et al., 2004; Ravn et al., 2005; Darbar et al., 2007; Juang et al., 2007). The studies identified two common polymorphisms, i.e., $\mathrm{Cx} 40-44 \mathrm{G} \rightarrow \mathrm{A}(\mathrm{rs} 35594137)$, and $\mathrm{Cx} 40+71$ $\mathrm{A} \rightarrow \mathrm{G}$ (at the transcription start site), and proposed that there was a greater representation of the $\mathrm{Cx} 40(-44 \mathrm{~A},+71 \mathrm{G})$ genotype as well as the respective allele frequencies in patients with AF (Groenewegen et al., 2003; Juang et al., 2007). Furthermore, a linkage relationship has been shown between these polymorphisms. The aim of the present study was to examine the gene polymorphism of Cx 40 (rs35594137) in patients with AF from the Uygur and Han populations, and controls, from the Xinjiang region to further define the correlation of $\mathrm{Cx} 40$ (rs35594137) with AF as well as whether racial and regional differences exist, and to provide a theoretical and scientific basis for in-depth analysis of the relationship between genetic polymorphism in Cx 40 (rs35594137) and AF.

\section{MATERIAL AND METHODS}

\section{Study participants}

The sample consisted of 244 individuals, aged 30-85 years, from the natural population with representation from the residential area in the more centralized population, economic 
development level, and medical conditions from Turpan city and Kashi city in Xinjiang; the cluster sampling method was utilized for collection. The sampling of the present study was taken with unequal probability at three states in different levels. The primary sampling units referred to village community in the street or township. The secondary sampling units referred to residential community and village community. The third sampling unit referred to each household where one person was chosen.

The people in the AF group consisted of patients who were clearly diagnosed with AF from the above-mentioned areas; and the people in the control group were those without evidence of AF in an echocardiogram (ECG, including Holter monitoring). There were 122 cases collected from the AF case group (58 Han and 64 Uygur). The cases of the control group were collected based on the matching conditions of ages and genders with the proportion of 1:1 corresponding to the ethnicity of the case group; that is, 58 Han and 64 Uygur individuals were included in the control group. The mean $( \pm \mathrm{SD})$ ages of the Han $\mathrm{AF}$ and control groups were $66.70 \pm 3.48$ and $65.23 \pm 2.35$ years, respectively. In the Uygur population, the mean ages in the AF and control groups were $64.53 \pm 2.35$ and $63.26 \pm 3.85$ years, respectively. The study was approved by the Ethics Committee of the People's Hospital of Xinjiang Uygur Autonomous Region and all the subjects had signed the informed consent form. In the study, recorded the general conditions of the chosen subjects as well as risk factors for the main common cardiovascular diseases and AF, including high pressure, coronary heart disease, smoking, and alcohol consumption, as well as ultrasonic left atrium diameter (LAD) and left ventricular ejection fraction (LVEF). Investigation methods and quality control were based on the investigation of cardiovascular diseases and epidemiology dominated by AF in the group with the unified design and unified proposal in the internationally common standardized investigation methods. The investigation covered examination by questionnaire and physical examination. The main contents of the questionnaire included: general condition, career, labor intensity, personal AF history, AF history of the family, smoking, drinking. The physical examination included: measurements of blood pressure, heart rate, ECG, height, weight, waistline, abdominal circumference and hipline. The criteria for enrollment were: 1) the present ECG showed $\mathrm{AF} ; 2$ ) the doctor had diagnosed the patient with AF and previous ECG of AF or Holter record were available; 3) ECG or Holter record that had been diagnosed to indicate AF was available; and 4) the diagnosis of high blood pressure was made according to the standard of Guide for Treatment of High Blood Pressure issued by World Health Organization (WHO)/International Society of Hypertension (ISH) in 1999.

\section{Study methods}

From each participant, $5 \mathrm{~mL}$ peripheral blood was taken and $0.5 \mathrm{M}$ anticoagulant (ethylenediaminetetraacetic acid) was added. The DNA in the white cells in the peripheral blood was abstracted with the high-salt precipitation method and was dissolved in TE tube for storage at $-20^{\circ} \mathrm{C}$. Ultraviolet spectrophotometry was used to determine the concentration of DNA in the specimen, which was diluted for further usage. For detection of polymorphic variants of $\mathrm{Cx}$ 40 rs35594137, the polymorphic gene section including Cx 40 rs35594137 was amplified by polymerase chain reaction (PCR) and then the genotype was determined with HaeIII restriction endonuclease analysis. For the single nucleotide change at $\mathrm{Cx} 40(\mathrm{rs} 35594137, \mathrm{G} \rightarrow \mathrm{A})$, the amplification primers were: positive-sense strand: 5'-CCC TCT TTT TAA TCG TAT CTG TGG C-3'; negative-sense strand: 5'-GGT GGA GGG AAG AAG ACT TTT AG-3'; the PCR primer 
product size was $150 \mathrm{bp}$. Following HaeIII restriction enzyme analysis, if the bases of site were -44 , then the restriction enzyme fragments were 126 and $24 \mathrm{bp}$, respectively. After PCR amplification, $5 \mu \mathrm{L}$ amplification product was electrophoresed using 1.5\% agarose gel and a 100-bp DNA size marker was used for comparison (Zhou et al., 2008) (Figure 1).

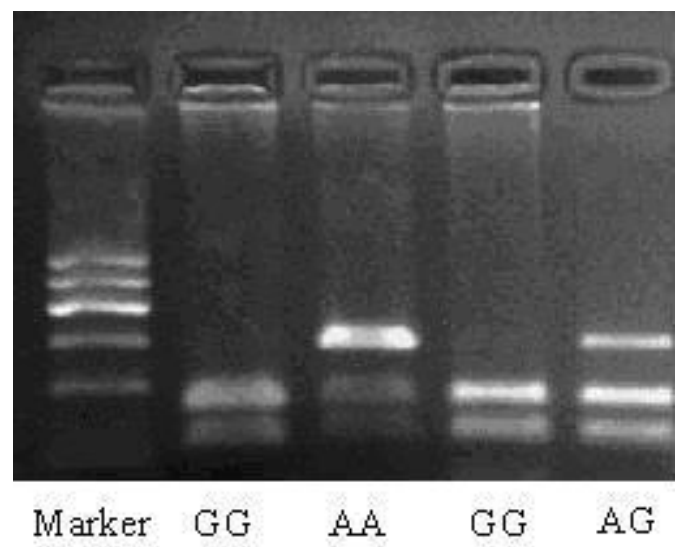

Figure 1. Electropherogram of polymerase chain reaction-restriction fragment length polymorphism fragments of the three connexin 40 genotypes.

\section{Statistical analysis}

The SPSS 17.0 software (SPSS, Chicago, IL, USA) was used to perform statistical analysis and the SHEsis software was used to detect the representation of the specimen groups under Hardy-Weinberg equilibrium and the distribution of the genotypes in each group. When Hardy-Weinberg equilibrium was realized, the measurement data in each group were represented by mean \pm standard deviation. The Student $t$-test was used for the comparison between two groups and the $\chi^{2}$ test was used for the comparison between the differences of genotype and allele frequencies between the two groups. For the comprehensive evaluation of the relevance between each factor and $\mathrm{AF}$, multiple-factor logistic regression analysis was performed. Differences were regarded to have significance when $\mathrm{P}<0.05$.

\section{RESULTS}

\section{General condition of the study participants}

In the Han cohort, the mean ages of the people in the patient and control groups were $65.5 \pm 4.38$ years; in the Uygur cohort, the mean ages of the people in the patient and control groups were $66.35 \pm 6.50$ years. Matching was made based on the ages and genders in the patient and control groups. In the Han and Uygur cohorts, the difference of smoking history and drinking in the patient and control groups had no statistical significance $(\mathrm{P}<0.05)$, while the history of coronary heart diseases and high blood pressure were significantly different $(\mathrm{P}$ $<0.05)$. In the Han and Uygur cohorts, all the LAD and LVEF values in the AF group were clearly higher than those in the control group and the differences were statistically significant $(\mathrm{P}<0.05$; Tables 1 and 2$)$. 
Table 1. General condition of study participants in the Han cohort (means \pm SD).

\begin{tabular}{lccc}
\hline & Patient group $(\mathrm{N}=58)$ & Controls $(\mathrm{N}=58)$ & $\mathrm{P}$ \\
\hline LAD $(\mathrm{mm})$ & $35.84 \pm 4.34$ & $29.65 \pm 5.65$ & $<0.01$ \\
LVEF $(\%)$ & $60.45 \pm 1.34$ & $66.69 \pm 2.32$ & $<0.01$ \\
Coronary heart diseases [N (\%)] & $40(62.5)$ & $26(46.63)$ & 0.042 \\
High blood pressure [N (\%)] & $43(74.14)$ & $9(15.52)$ & $<0.01$ \\
Smoking [N (\%)] & $31(53.45)$ & $28(48.28)$ & 0.314 \\
Alcohol consumption [N (\%)] & $15(25.86)$ & $10(17.24)$ & 0.219 \\
\hline
\end{tabular}

$\mathrm{LAD}=$ left atrium diameter; $\mathrm{LVEF}=$ left ventricular ejection fraction.

Table 2. General condition of study participants in the Uygur cohort (means \pm SD).

\begin{tabular}{lccc}
\hline & Case group $(\mathrm{N}=64)$ & Controls $(\mathrm{N}=64)$ & $\mathrm{P}$ \\
\hline LAD (mm) & $37.36 \pm 2.36$ & $30.28 \pm 1.34$ & $<0.01$ \\
LVEF (\%) & $62.76 \pm 4.34$ & $66.21 \pm 2.33$ & $<0.01$ \\
Coronary heart diseases [N (\%)] & $39(60.94)$ & $20(31.25)$ & 0.021 \\
High blood pressure [N (\%)] & $47(73.44)$ & $19(29.69)$ & $<0.01$ \\
Smoking [N (\%)] & $26(40.63)$ & $23(35.94)$ & 0.061 \\
Alcohol consumption [N (\%)] & $30(46.88)$ & $34(53.13)$ & 0.170 \\
\hline
\end{tabular}

$\mathrm{LAD}=$ left atrium diameter; $\mathrm{LVEF}=$ left ventricular ejection fraction.

\section{Analysis of the results of the patient and control groups of the Han and Uygur cohorts}

In the Han cohort, 30, 16, and 12 patients with $\mathrm{AF}$ carried the genotypes of $\mathrm{AA}, \mathrm{AG}$, and GG, respectively, reflecting 51.72, 27.59, and $20.69 \%$, respectively, of the affected group; there were 27,15 , and 16 controls with genotypes AA, AG, and GG, respectively, reflecting 46.55, 25.86, and $27.59 \%$, respectively, of the control group. Compared with those in the patient and control groups, the differences of the three genotypes of AA, AG, and GG had no statistical significance $\left(\chi^{2}=1.237, P=0.56\right)$. In the patient group, the allele frequencies of $A$ and $G$ were 72 and 44 cases, accounting for 62.07 and $37.93 \%$, respectively. In the control group, the allele frequencies of A and $\mathrm{G}$ were 67 and 49 cases, accounting for 57.59 and $42.24 \%$, respectively, and the distribution differences had no statistical significance $\left(\chi^{2}=0.006, \mathrm{P}=0.86\right.$; Table 3$)$.

Table 3. rs35594137 genotype distribution and allele frequency (\%) in the Han cohort.

\begin{tabular}{|c|c|c|c|c|c|c|c|c|c|}
\hline & \multicolumn{3}{|c|}{ Genotype } & \multirow[t]{2}{*}{$\chi^{2}$} & \multirow[t]{2}{*}{$\mathrm{P}$} & \multicolumn{2}{|c|}{ Allele } & \multirow[t]{2}{*}{$\chi^{2}$} & \multirow[t]{2}{*}{$\mathrm{P}$} \\
\hline & AA & $\mathrm{AG}$ & GG & & & A & G & & \\
\hline $\begin{array}{l}\text { Case group } \\
\text { Controls }\end{array}$ & $\begin{array}{l}30(51.72) \\
27(46.55)\end{array}$ & $\begin{array}{l}16(27.59) \\
15(25.86)\end{array}$ & $\begin{array}{l}12(20.69) \\
16(27.59)\end{array}$ & 1.237 & 0.56 & $\begin{array}{l}72(62.07) \\
67(57.59)\end{array}$ & $\begin{array}{l}44(37.93) \\
49(42.24)\end{array}$ & 0.006 & 0.86 \\
\hline
\end{tabular}

In the Uygur cohort, 35, 16, and 13 patients with $\mathrm{AF}$ carried the genotype of AA, AG, and GG, respectively, reflecting 54.60, 25.00, and 20.31\%, respectively, of the affected group; there were 25, 20, and 19 controls with the genotype of AA, AG, and GG, respectively, reflecting $39.06,30.77$, and $29.69 \%$, respectively, of the control group. The differences of the three genotypes of AA, AG, and GG showed statistical significance between the patient and control groups $\left(\chi^{2}=8.244, \mathrm{P}=0.012\right)$. In the $\mathrm{AF}$ group, the allele frequencies of $\mathrm{A}$ and $\mathrm{G}$ were 80 and 48 cases, accounting for 62.50 and $37.50 \%$, respectively. In the control group, the allele frequencies of A and $\mathrm{G}$ were 70 and 58 cases, accounting for 54.69 and $45.31 \%$, respectively, and the distribution differences had statistical significance $\left(\chi^{2}=8.120, P=0.032\right.$; Table 4$)$. 
Table 4. rs35594137 genotype distribution and allele frequency (\%) in the Uygur cohort.

\begin{tabular}{|c|c|c|c|c|c|c|c|c|c|}
\hline & \multicolumn{3}{|c|}{ Genotype } & \multirow[t]{2}{*}{$\chi^{2}$} & \multirow[t]{2}{*}{ P } & \multicolumn{2}{|c|}{ Allele } & \multirow[t]{2}{*}{$\chi^{2}$} & \multirow[t]{2}{*}{$P$} \\
\hline & AA & $\mathrm{AG}$ & GG & & & A & G & & \\
\hline $\begin{array}{l}\text { Case group } \\
\text { Controls }\end{array}$ & $\begin{array}{l}35(54.69) \\
25(39.06)\end{array}$ & $\begin{array}{l}16(25.00) \\
20(30.77)\end{array}$ & $\begin{array}{l}13(20.31) \\
19(29.69)\end{array}$ & 8.244 & 0.012 & $\begin{array}{l}80(62.50) \\
70(54.69)\end{array}$ & $\begin{array}{l}48(37.50) \\
58(45.31)\end{array}$ & 8.120 & 0.032 \\
\hline
\end{tabular}

\section{Analysis of the correlation between the $\mathrm{Cx} 40$ (rs35594137) polymorphism and AF in the Han and Uygur}

After multifactor logistic regression analysis was applied to control for the potential confounding factors of coronary heart diseases, high blood pressure, smoking, and alcohol consumption, the difference of $\mathrm{Cx} 40$ (rs35594137) between the patient and control groups had no statistical significance in the Han, and thus the polymorphism of $\mathrm{Cx} 40$ (rs35594137) had no relationship with AF. Excluding the influences of other factors of smoking, alcohol consumption, and genes, high blood pressure and coronary heart diseases were considered risk factors for $\mathrm{AF}(\mathrm{P}<0.05$; Table 5).

\section{Table 5. Multiple-factor logistic regression analysis.}

\begin{tabular}{|c|c|c|c|c|c|c|}
\hline \multirow[t]{2}{*}{ Risk factors } & \multicolumn{3}{|c|}{ Han people } & \multicolumn{3}{|c|}{ Uygur people } \\
\hline & $\chi^{2}$ & OR & $P$ & $\chi^{2}$ & OR & $P$ \\
\hline Coronary heart diseases & 14.87 & 2.346 & $<0.01$ & 12.65 & 1.784 & $<0.01$ \\
\hline High blood pressure & 16.70 & 2.375 & $<0.01$ & 15.25 & 1.867 & $<0.01$ \\
\hline Smoking & 3.68 & 1.250 & 0.12 & 3.87 & 1.123 & 0.12 \\
\hline Alcohol consumption & 3.88 & 1.420 & 0.15 & 4.55 & 1.376 & 0.13 \\
\hline rs35594137 (AA) & 5.40 & 1.023 & 0.65 & 10.80 & 1.654 & $<0.01$ \\
\hline
\end{tabular}

$\mathrm{OR}=$ odds ratio.

After multifactor logistic regression analysis was applied to control the potential confounding factors of coronary heart diseases, high blood pressure, smoking, and alcohol consumption, the difference of $\mathrm{Cx} 40$ (rs35594137) between the patient and control groups had statistical significance in the Uygur cohort $(\mathrm{P}<0.05)$. In the Uygur cohort, the genotype AA of Cx 40 (rs35594137) was one of the independent risk factors for the Uygur patients with AF. Of the other factors of smoking, alcohol consumption, and genes, high blood pressure and coronary heart diseases were considered risk factors for $\mathrm{AF}$ in Uygur cohort $(\mathrm{P}<0.05$; Table 5).

\section{DISCUSSION}

In recent years, the gap junction closely related to the occurrence of $\mathrm{AF}$ and the role of $\mathrm{Cx} 40$ in $\mathrm{AF}$ has been given more attention. $\mathrm{Cx} 40$ is the most important coupled protein of the atrial cytokines. Jiang et al. (2012) studied the expression and distribution of disorders in patients with AF and found that these changes can lead to obstacles in electric coupling, abnormal conductance, as well as the occurrence of reentrance between neighboring myocardial cells, and thus may be one of the pathological mechanisms of AF.

van der Velden et al. (2000) found that the expression of $\mathrm{Cx} 40$ in the atrial tissue of goats with $\mathrm{AF}$ was reduced. The $\mathrm{Cx} 40$ in the atrial tissue under chronic AF was expressed 
following local damage, caused by disordered electrical conductance leading to the occurrence of AF. Polontchouk et al. (2001) studied the chronic AF and the auricula dextra of the control people and found that the expression of $\mathrm{Cx}$ increased by 2.7-fold. Research showed that gene mutation of $\mathrm{Cx} 40$ in human hearts caused the reduction of $\mathrm{Cx} 40$ protein expression and could result in the development of AF (Wirka et al., 2011), while in animal experiments, the transfection connexin genes in pig heart could improve the conductance and effectively reduce the occurrence of AF (Igarashi et al., 2012). At present, it is held that the impedance and conductance speed between neighboring atrial tissues are clearly different due to the high homogeneity in the distribution of $\mathrm{Cx} 40$ in the heterogeneity in human muscles, which will be easy to form the reentrant loop for the AF; thus, the transcription and upregulation of $\mathrm{Cx}$ 40 in the atrial muscles is an important factor to promote the occurrence and duration of AF (Dupont et al., 2001). Juang et al. (2007) coded Cx protein genes and discovered two interlocking polymorphic sites, $\mathrm{Cx} 40(-44 \mathrm{~A},+71 \mathrm{G})$ and $\mathrm{Cx} 40(-44 \mathrm{G},+71 \mathrm{~A})$, and found that $\mathrm{Cx}$ $40(-44 \mathrm{~A},+71 \mathrm{G})$ was related to the occurrence of AF. In China, Zhou et al. (2008) showed that the genotype frequency AA and allele frequency AA were higher at site - 44 of $\mathrm{Cx} 40$ in $\mathrm{AF}$ group than those in the non-AF group $(\mathrm{P}<0.05)$; thus, the polymorphism of $\mathrm{Cx} 40(-44$ $\mathrm{G} \rightarrow \mathrm{A}$ ) may have associations with the occurrence of AF. As the differences of the allele frequency and genotype distribution of rs 35594137 between the patient and control groups in the Uygur cohort had a statistical significance, the results of the present study suggested that $\mathrm{Cx}$ 40 (rs35594137) is related to the occurrence of AF. The distribution of Cx 40 (rs35594137) in patients with AF may vary due to race and regions. The AA allele frequency in the control group of Han people was $42.24 \%$, while that in the Uygur people was $45.31 \%$, presenting slight variation. The results pointed out that the differences of racial hereditary structure can be reflected from this polymorphic site; however, positive errors may exist because of small sample number and inefficient statistical efficiency. There are multiple risk factors of coronary heart disease, including high blood pressure, smoking, and alcohol consumption, among others. In this study, Cx 40 (rs35594137) had no association with AF in the Han cohort when the confounding factors were examined under multiple-factor logistic regression analysis, whereas Cx 40 (rs35594137) appeared to be an independent risk factor for the occurrence of AF in the Uygur cohort. In recent years, studies in China and abroad showed that single nucleotide polymorphisms of different genes can induce the occurrence of AF; thus, AF was predicted to be a kind of polygenic disease. The results of the present study suggest that $\mathrm{Cx}$ 40 (rs35594137, $-44 \mathrm{G} \rightarrow \mathrm{A}$ ) can be used as one of the markers of risks of the occurrence of AF in the Uygur people. Following the continuous developments in science and technology, it is expected that more and more gene polymorphism sites will be found to be related to AF.

The study provides a theoretical basis for further research on the susceptibility of AF in different nations to determine the molecular biological mechanism of AF, and provides evidence for the relevance between $\mathrm{Cx} 40$ (rs35594137) and AF among different nations. These results will be useful for the formulation of corresponding gene diagnosis strategies for the mutation variance of different ethnicities, and for providing the theoretical basis for individual therapy of AF in regional groups.

\section{ACKNOWLEDGMENTS}

Research supported by grant \#2012211A091 from the Natural Science Foundation of Xinjiang Uygur Autonomous Region. 


\section{REFERENCES}

Darbar D, Motsinger AA, Ritchie MD, Gainer JV, et al. (2007). Polymorphism modulates symptomatic response to antiarrhythmic drug therapy in patients with lone atrial fibrillation. Heart Rhythm. 4: 743-749.

Dupont E, Ko Y, Rothery S, Coppen SR, et al. (2001). The gap-junctional protein connexin40 is elevated in patients susceptible to postoperative atrial fibrillation. Circulation 103: 842-849.

Groenewegen WA, Firouzi M, Bezzina CR, Vliex S, et al. (2003). A cardiac sodium channel mutation cosegregates with a rare connexin40 genotype in familial atrial standstill. Circ. Res. 92: 14-22.

Igarashi T, Finet JE, Takeuchi A, Fujino Y, et al. (2012). Connexin gene transfer preserves conduction velocity and prevents atrial fibrillation. Circulation 125: 216-225.

Jiang CY, Ling L and Fang CM (2012). The alteration of connexin 40 distribution and expression in right atrial myocardium in patients with different types of atrial fibrillation. Chin. J. Arterioscler. 6: 508-513.

Juang JM, Chern YR, Tsai CT, Chiang FT, et al. (2007). The association of human connexin 40 genetic polymorphisms with atrial fibrillation. Int. J. Cardiol. 116: 107-112.

Lai LP, Su MJ, Yeh HM, Lin JL, et al. (2002). Association of the human minK gene $38 \mathrm{G}$ allele with atrial fibrillation: evidence of possible genetic control on the pathogenesis of atrial fibrillation. Am. Heart J. 144: 485-490.

Polontchouk L, Haefliger JA, Ebelt B, Schaefer T, et al. (2001). Effects of chronic atrial fibrillation on gap junction distribution in human and rat atria. J. Am. Coll. Cardiol. 38: 883-891.

Ravn LS, Hofman-Bang J, Dixen U, Larsen SO, et al. (2005). Relation of 97T polymorphism in KCNE5 to risk of atrial fibrillation. Am. J. Cardiol. 96: 405-407.

Schreieck J, Dostal S, von Beckerath N, Wacker A, et al. (2004). C825T polymorphism of the G-protein beta3 subunit gene and atrial fibrillation: association of the TT genotype with a reduced risk for atrial fibrillation. Am. Heart J. 148: 545-550.

van der Velden HM, Ausma J, Rook MB, Hellemons AJ, et al. (2000). Gap junctional remodeling in relation to stabilization of atrial fibrillation in the goat. Cardiovasc. Res. 46: 476-486.

Wirka RC, Gore S, Van Wagoner DR, Arking DE, et al. (2011). A common connexin-40 gene promoter variant affects connexin-40 expression in human atria and is associated with atrial fibrillation. Circ. Arrhythm. Electrophysiol. 4: 87-93.

Zhou Q, Zhang P and Li X (2008). The study on the association between human connexin 40 gene polymorphism and atrial fibrillation. J. Clin. Electrocardiol. 17: 204-206. 